\title{
Transcriptional variation and divergence of host-finding behaviour in Steinernema carpocapsae infective juveniles
}

\author{
Neil D. Warnock ${ }^{\dagger}$, Deborah Cox ${ }^{\dagger}$, Ciaran McCoy, Robert Morris and Johnathan J. Dalzell ${ }^{*}$
}

\begin{abstract}
Background: Steinernema carpocapsae is an entomopathogenic nematode that employs nictation and jumping behaviours to find potential insect hosts. Here we aimed to investigate the transcriptional basis of variant hostfinding behaviours in the infective juvenile (IJ) stage of three S. carpocapsae strains (ALL, Breton and UK1), with a focus on neuronal genes known to influence behaviour in other nematode species. Identifying gene expression changes that correlate with variant host-finding behaviours will further our understanding of nematode biology.

Results: RNA-seq analysis revealed that whilst up to $28 \%$ of the S. carpocapsae transcriptome was differentially expressed $(P<0.0001)$ between strains, remarkably few of the most highly differentially expressed genes ( $>2 \log 2$ fold change, $P<0.0001)$ were from neuronal gene families. $S$. carpocapsae Breton displays increased chemotaxis toward the laboratory host Galleria mellonella, relative to the other strains. This correlates with the up-regulation of four srsx chemosensory GPCR genes, and a sodium transporter gene, asic-2, relative to both ALL and UK1 strains. The UK1 strain exhibits a decreased nictation phenotype relative to ALL and Breton strains, which correlates with co-ordinate up-regulation of neuropeptide like protein 36 ( $(\mathrm{p} / \mathrm{p}-36)$, and down-regulation of an srt family GPCR gene, and a distinct asic-2-like sodium channel paralogue. To further investigate the link between transcriptional regulation and behavioural variation, we sequenced microRNAs across IJS of each strain. We have identified 283 high confidence microRNA genes, yielding 321 predicted mature microRNAs in S. carpocapsae, and find that up to $36 \%$ of microRNAs are differentially expressed $(P<0.0001)$ between strains. Many of the most highly differentially expressed microRNAs ( $>2 \log 2$ fold, $P<0.0001$ ) are predicted to regulate a variety of neuronal genes that may contribute to variant host-finding behaviours. We have also found evidence for differential gene isoform usage between strains, which alters predicted microRNA interactions, and could contribute to the diversification of behaviour.
\end{abstract}

Conclusions: These data provide insight to the transcriptional basis of behavioural variation in S. carpocapsae, supporting efforts to understand the molecular basis of complex behaviours in nematodes.

Keywords: Nematode, Transcriptome, microRNA, Isoform

\section{Background}

Parasitic nematodes employ a variety of behaviours that maximise opportunity for host contact and invasion. These behaviours vary across species, ranging from passive reliance on host ingestion, through pro-active host-finding by migration, nictation, and even jumping [1-3]. Each of these behavioural strategies rely on the incorporation of multiple

\footnotetext{
*Correspondence: j.dalzell@qub.ac.uk

${ }^{+}$Neil D. Warnock and Deborah Cox contributed equally to this work.

School of Biological Sciences, Queen's University Belfast, Belfast, Northern Ireland
}

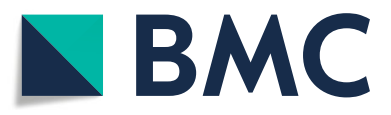

(๑) The Author(s). 2019 Open Access This article is distributed under the terms of the Creative Commons Attribution 4.0 International License (http://creativecommons.org/licenses/by/4.0/), which permits unrestricted use, distribution, and reproduction in any medium, provided you give appropriate credit to the original author(s) and the source, provide a link to the Creative Commons license, and indicate if changes were made. The Creative Commons Public Domain Dedication waiver (http://creativecommons.org/publicdomain/zero/1.0/) applies to the data made available in this article, unless otherwise stated.

sensory inputs, spanning chemosensory, olfactory, mechanosensory, thermosensory and hygrosensory circuits $[2,4]$. Nematode host-finding strategies are also remarkably plastic, varying in response to experience and environment [5]. Despite the obvious importance of parasite host-finding behaviour to medical, veterinary and agricultural interests, we know relatively little about the genes involved in regulating these behaviours. It may be possible to develop new approaches to parasite control by targeting components of the parasite host-finding apparatus. 
The evident complexity of nematode behaviour belies their relative neuroanatomical simplicity. It is thought that the neurochemical complexity of nematodes is central to their diverse behavioural capacity and adaptability [6]; neuropeptides in particular are highly enriched and conserved between nematode species with diverse life history traits [7]. It has also been demonstrated that FMRFamide-like peptide $(f l p)$ genes are co-ordinately up-regulated in host-finding stages of diverse parasitic nematodes [8], and that they contribute to various host-finding behaviours [8,9], along with insulin-like peptides [10] and neuropeptide-like proteins [11]. Diverse neuronal gene families also contribute to the surprising behavioural enrichment of such simple organisms [12]. Variation in gene transcript abundance and sequence identity is central to the phenotypic plasticity of cells, tissues and organisms, underpinning behavioural variation.

Small non-coding RNAs also contribute to gene regulation, as a factor of developmental stage, and in response to the environment. Small RNAs have been implicated in driving phenotypic novelty and adaptation within and between species [13-16]. MicroRNAs are small non-coding RNAs that negatively regulate target gene expression across higher organisms [17], and have been shown to modulate neuronal connectivity, synaptic remodelling [18] and memory within the olfactory system of other invertebrates [19]. In vivo cross-linking and immunoprecipitation of microRNA-specific argonaute proteins coupled with sequencing of bound mRNA transcripts in Caenorhabditis elegans, demonstrates an enrichment of neuronal gene families. This confirms that many neuronal genes with known involvement in behaviour are biologically relevant microRNA targets [20]. Key to understanding the contribution of neuronal gene function and microRNA regulation to host-finding behaviour in parasitic nematodes is the development of a suitable model system through provision of foundational datasets.

Steinernema spp. nematodes are obligate entomopathogens that invade and kill insect hosts through coordinated action with commensal Xenorhabdus bacteria [21]. Steinernema infective juveniles (IJs) display qualitatively different host-finding strategies between species [22], representing a unique resource for the comparative analysis of behaviour. Steinernema carpocapsae is generally considered to employ an 'ambushing' strategy, characterised by nictation and jumping behaviours. Nictation is enacted by nematodes that stand upright, waving their anterior in the air [23]. During nictation, the nematode can respond to sensory stimuli in one of three ways: (i) it can cease nictation and transition to a migratory phase; (ii) it can engage in a torpid 'standing' phenotype that may enhance opportunity for host attachment; and (iii) it may jump directionally in response to volatile and mechanosensory input $[8,23]$. Whilst the jumping behaviour is thought to be unique to a small number of Steinernema spp., nictation is shared amongst many economically important animal parasitic nematodes, alongside the model nematode $C$. elegans, for which nictation represents a long-range phoretic dispersal behaviour that is regulated by IL2 neurons [24]. In this study our aim was to profile the host-finding behaviours of $S$. carpocapsae strains, and to identify protein-coding and noncoding RNAs that are differentially expressed in strains with variant behaviours. These data will underpin future efforts to understand nematode host-finding behaviour.

\section{Methods}

\section{S. carpocapsae culture}

Steinernema carpocapsae strains (ALL, Breton and UK1) were maintained in Galleria mellonella at $23^{\circ} \mathrm{C}$. S. carposapsae strains were gifted by Prof Ali Mortazavi (University of California, Irvine), Prof Nelson Simões (University of the Azores, Portugal), and BASF UK, respectively. IJs were collected by White trap [25] in a solution of Phosphate Buffered Saline (PBS). Freshly emerged IJs were used for each experiment. Individual biological replicates and RNAseq libraries described below were derived from a mixed population of IJs that emerged from multiple $G$. mellonella cadavers on the same day.

\section{Behavioural assays}

Galleria mellonella host-finding assays and dispersal assays were conducted as published [9]; in both instances five biological replicates were assayed across three technical replicates each. A chemosensory index (CI) was calculated following host-finding assays, giving a measure of relative attraction for participating IJs [8]. For the nictation assays, micro-dirt chips were made from a PDMS mould [26], with $3.5 \% \mathrm{ddH}_{2} \mathrm{O}$ agar. $20 \mathrm{IJs}$ suspended in 1.5 $\mu \mathrm{l}$ PBS were pipetted onto the micro-dirt chip, under a binocular light microscope. Once the liquid had evaporated and the IJs could move freely, the number of nictating IJs was counted at 1, 2.5 and 5 min intervals. Nictation assays were conducted over five biological replicates, each with five technical replicates of 20 IJs each. Each dataset was assessed by Brown-Forsythe and Bartlett's tests to examine homogeneity of variance between groups. One way ANOVA and Tukey's multiple comparison tests were then used to assess statistically significant differences in mean across experimental groups. Tests were conducted in Graphpad Prism 7.02.

\section{RNA-seq, differential expression and isoform variant analysis}

Six biological replicates of each strain were prepared from approximately 10,000 individuals $(80 \mu \mathrm{l}$ packed volume after centrifugation at $2000 \mathrm{rpm}$ for $2 \mathrm{~min}$ ) each. 
Total RNA was extracted from IJs using TRIzol Reagent (Invitrogen) and DNase treated using the Turbo DNase kit (Ambion) following manufacturer's instructions. RNA quantity were assessed by gel electrophoresis and quantified using Qubit RNA BR Assay Kit (Life Technologies) as per manufacturer's instructions. A total of six transcriptome libraries (150 bp, paired end) were prepared for each $S$. carpocapsae strain, from $1 \mu \mathrm{g}$ of total RNA each, using the TruSeq RNA Library Prep Kit v2 (Illumina) following manufacturer's instructions. Sequencing was performed on the HiSeq2500 instrument. Fastq files were assessed for quality using the FastQC (v. 0.11.3) package [27]. Adapters, low quality bases, and reads shorter than 36 bp were removed using the Trimmomatic (v. 0.35) package [28]. The trimmed data quality was then re-assessed using the FastQC package. The $S$. carpocapsae genomic contigs (PRJNA202318.WBPS9) and associated GFF file (PRJNA202318.WBPS9) were downloaded from https:// parasite.wormbase.org/index.html. Annotations were converted to GTF format using Cufflinks (v. 2.2.2.20150701) [29]. High quality reads were then mapped to the S. carpocapsae genome [30] using the STAR (v. 2.5.3a) package [31]. Isoform expression levels were quantified using the RSEM (v. 1.2.19) package [32], and integrated EBseq package [33]. Raw read counts mapping to each gene in each sample were consolidated into a single count table. This process was repeated for each isoform. Downstream analyses was performed using R (v. 3.3.1) [34]. Differential expression of genes was quantified using the DESeq2 (v. 1.14.1) package [35]. Graphics were generated in R using RColorBrewer (v. 1.1-2) [36], gplots (v. 3.0.1) [37], geneplotter (v. 1.52) [38] and pheatmap (v. 1.0.8) [39] packages with custom $\mathrm{R}$ scripts. Additionally, we conducted a GO enrichment analysis of differentially expressed genes that correlated with behavioural variation between strains, using g:Profiler (https://biit.cs. ut.ee/gprofiler/gost), using standard parameters.

\section{MicroRNA sequencing, discovery and quantification}

Small RNA libraries were generated from the same RNA samples that were used for matched transcriptomes. A total of six small RNA libraries were prepared for each strain, from $1 \mu \mathrm{g}$ of total RNA each, using the TruSeq Small RNA library Kit (Illumina) following manufacturer's instructions. $50 \mathrm{bp}$ single-end libraries were sequenced on the HiSeq2500 instrument. Fastq files were assessed for quality using the FastQC (v. 0.11.3) package [27]. Adapters, low quality bases, and reads shorter than 13 bp were removed using the Cutadapt package (v. 1.8) [40]. Sequence reads without adapters were also discarded. Reads that passed QC were mapped to the genome sequence of $S$. carpocapsae, and microRNAs were identified by miRDeep2 (v. 2.0.0.8) [41], using a training set of mature and precursor microRNA sequences downloaded from miRBase (http://www.mirbase.org/). Naming of microRNAs was preferentially aligned with C. elegans, as indicated by miRDeep 2 output. Novel $S$. carpocapsae microRNAs were named and numbered sequentially, taking care to avoid overlap with any $C$. elegans microRNA. Differentially expressed microRNAs were identified as above, using the DESeq2 package, and were presented using RColorBrewer, gplots, geneplotter and pheatmap packages.

\section{MicroRNA target gene prediction}

Three and five prime UnTranslated Regions (UTRs) of computationally predicted $S$. carpocapsae genes were exported from wormbase parasite [30, 42] using the biomart function. Retrieved sequences were converted to fasta format and predicted microRNA binding sites were identified using miRanda [43]. Two separate miRanda analyses were performed, using i) default settings and ii) strict settings that require perfect conservation of seed site sequence complementarity between microRNA and target mRNA. Experimental verification of microRNA target predictions indicate that perfect complementarity between the microRNA seed region and target mRNA provides the highest degree of specificity and sensitivity [44]. However, Argonaute CLIP-seq analyses indicate that around $40 \%$ of all microRNA-mRNA interactions lack perfect seed region complementarity [45]; limiting analyses to perfect seed region requirements will lead to a substantial number of false negatives. MiRanda allows analyses that span canonical seed region complementarity, and non-canonical interactions, providing a robust overview of interactions that follow experimentally validated examples [44]. In each instance, we have included information of relative target site predictions using both strict and default target identification approaches.

\section{Annotation of neuropeptide, neurotransmitter, GPCR, innexin and ion channel genes}

S. carpocapsae neuropeptide gene orthologues were identified via reciprocal BLAST analysis. A list of available $C$. elegans FLP, NLP and INS pre-propeptide sequences were obtained from Wormbase [46] and used as BLASTp and tBLASTn search strings via the Wormbase ParaSite BLAST server [42] under default settings. The protein sequences for overlapping genes associated with each high scoring pair were then employed as BLASTp search strings against the available C. elegans protein dataset via the Wormbase BLAST server [46]. Where no overlapping gene annotation was available, novel predicted proteins were generated by concatenating high-scoring return sequences to facilitate reciprocation [47]. The top reciprocal BLAST hit from C. elegans was used to assign putative neuropeptide gene names, and subsequent manual comparison of $S$. carpocapsae neuropeptide primary sequences 
to established nematode neuropeptide motifs [48] was used to confirm or reassign gene names where appropriate. GPCR, ion channel and innexin genes were exported from Wormbase parasite according to GO term, using the biomart function. All neurotransmitter genes were identified by reciprocal BLAST, as above. Additionally, the identity of each gene represented in heatmaps and tables of this manuscript was confirmed by reciprocal BLAST, as above. In a number of instances, different $S$. carpocapsae genes reciprocated to the same $C$. elegans gene. In any such case, a simple numbering system was applied to gene names in order to reflect clustered identity.

\section{Results}

\section{Behavioural variation across S. carpocapsae strains}

$S$. carpocapsae Breton demonstrated an increased chemotaxis index in response to G. mellonella volatiles, indicating that significantly more IJs migrated toward the larvae relative to other strains (Fig. 1a; $0.88 \pm 0.009$, relative to $0.43 \pm$ 0.06 for ALL, and $0.31 \pm 0.08$ for UK1, $\left.P<0.0001^{* * * *}\right)$. $S$. carpocapsae UK1 exhibited a reduced nictation phenotype relative to the other strains (Fig. 1b; $2.26 \% \pm 0.99$ of UK1 IJs were nictating at the $5 \mathrm{~min}$ time point, relative to $26.5 \% \pm$ 3.8 of ALL strain IJs, and $27.7 \% \pm 3.7$ of Breton strain IJs,
$\left.P<0.0001^{* * * \%}\right)$. No statistically significant difference was observed in the dispersal behaviour of IJs from S. carpocapsae strains (Fig. 1c).

\section{Transcriptional variation across strains}

$7494(28 \%)$ and $3662(13.7 \%)$ of S. carpocapsae UK1 genes were differentially expressed $\left(\mathrm{P}<0.0001^{* * * * *}\right)$ relative to Breton and ALL strains, respectively (Fig. 2; Additional file 1). 4762 (17.7\%) of $S$. carpocapsae Breton genes were differentially expressed $\left(P<0.0001^{* * * * *}\right)$ relative to the ALL strain (Fig. 2). GO term enrichment revealed that transmembrane transport was the most statistically enriched gene classification when considering both enhanced chemotaxis (Breton relative to All and UK1) and decreased nictation (UK1 relative to All and Breton). Several additional statistically significant enrichments suggested a contribution from neuronal gene families (Additional file 1).

$22(0.08 \%)$ of the 1509 (5.3\%) most highly differentially expressed genes across all pairwise strain comparisons ( $>2 \log 2$ fold, $P<0.0001^{* * * * * *}$ ) were representative of neuronal gene families, based on GO term annotations (Additional file 1). Of these 22 neuronal genes, only eight were observed to share differential expression

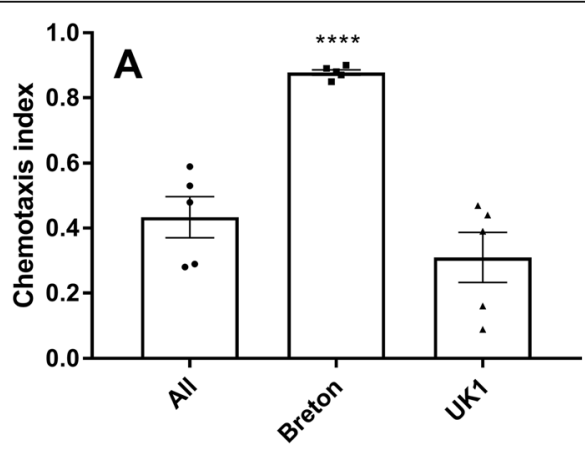

S. carpocapsae strain

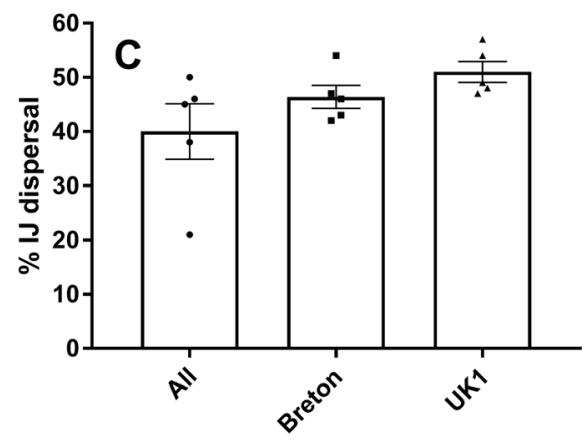

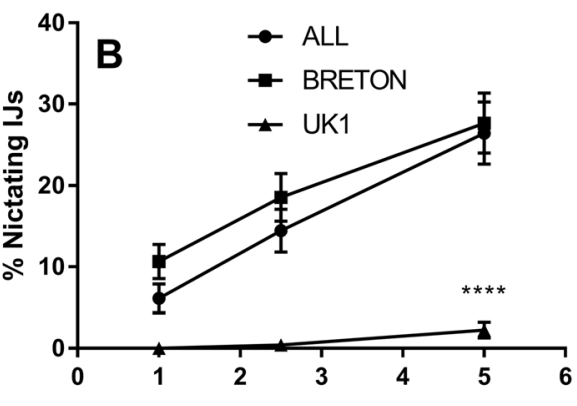

Minutes

S. carpocapsae strain

Fig. 1 S. carpocapsae strains display variant host-finding behaviours. a Mean chemotaxis index of S. carpocapsae strains in response to Galleria mellonella larvae; b Mean number of nictating S. carpocapsae IJs over a time-course; c Mean percentage dispersal of S. carpocapsae IJs into the peripheral assay zone (dispersed) after a $1 \mathrm{~h}$ timecourse. Data points represent mean \pm SEM; assessed by ANOVA and Tukey's multiple comparison test using Graphpad Prism 7.02; $P<0.0001^{* * * *}$ 


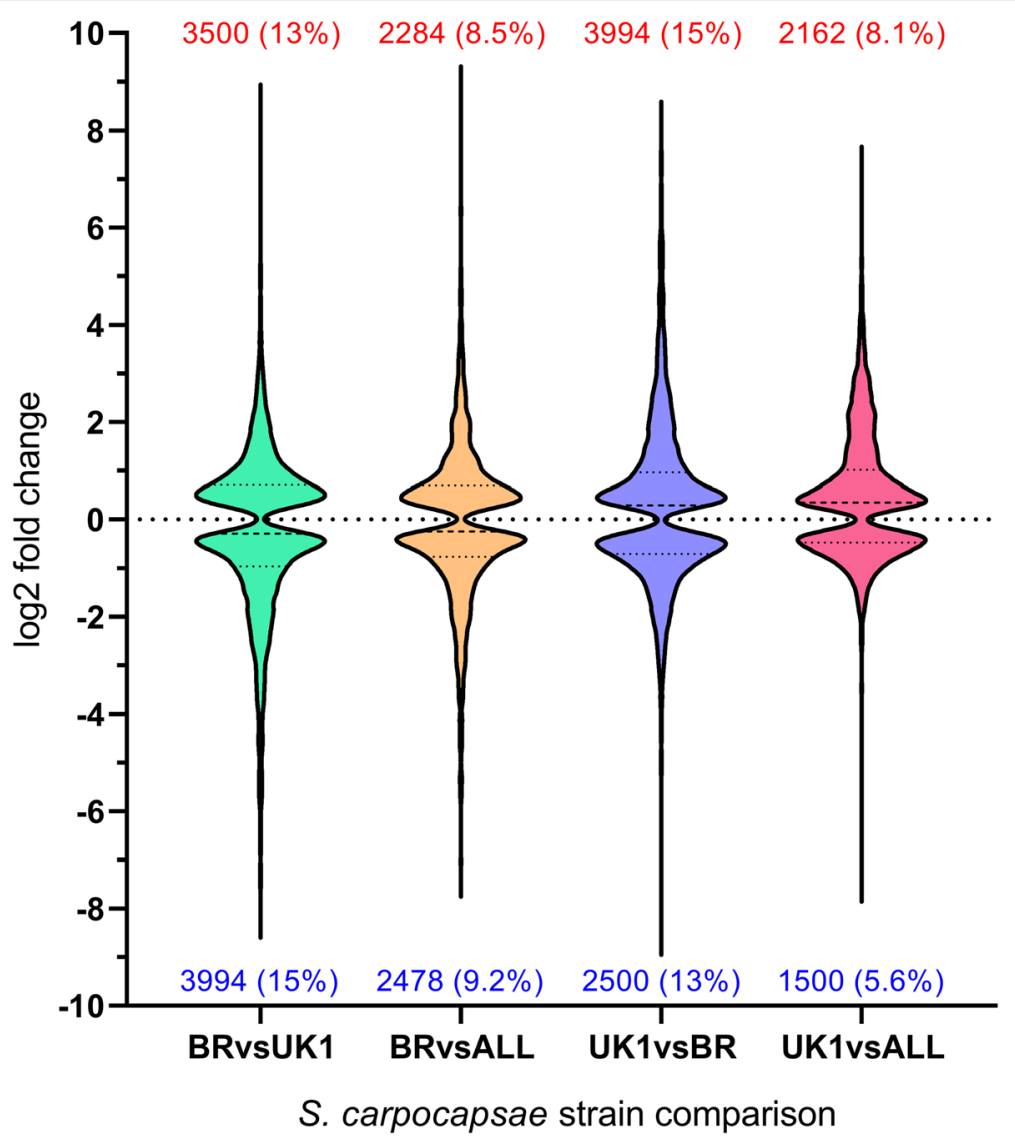

Fig. 2 Violin plot showing significantly up-regulated and down-regulated genes across pairwise S. carpocapsae strain comparisons. Statistically significant $\left(P<0.0001^{* * * *}\right)$ differentially expressed genes are plotted irrespective of log 2 fold change. Total number and relative percentage of up-regulated and down-regulated genes are presented above and below each plot, respectively

patterns across pairwise comparisons that correlate with distinct IJ behaviours; either increased chemotaxis (S. carpocapsae Breton; Fig. 1a) or decreased nictation (S. carpocapsae UK1; Fig. 1b). Specifically, shared up or down-regulation in S. carpocapsae Breton relative to both ALL and UK1 strains would correlate gene differential expression with increased chemotaxis to G. mellonella (Fig. 1a). Conversely, shared up or down-regulation in S. carpocapsae UK1 relative to both Breton and ALL strains would correlate gene differential expression with reduced nictation behaviour (Fig. 1b). In order to explore the transcriptional regulation of neuronal gene families implicated in these behavioural differences, we assessed each gene family represented in the highly differentially expressed category, as defined above. This encompassed G-protein coupled receptors (GPCRs), ion channels, innexins, neuropeptides and neurotransmitter synthesis, degradation and transport genes (Additional file 1).

\section{Neuropeptide and neurotransmitter genes}

$N l p-36$ was the only neuropeptide-like protein gene to demonstrate significant fold change differences $(>2 \log 2$ fold, $\left.P<0.0001^{* * * *}\right)$ that correlate with decreased nictation behaviour in $S$. carpocapsae UK1 across pairwise comparisons (up-regulated 2.8 and $3.5 \log 2$ fold, $P<0.0001^{* * * * *}$ relative to Breton and ALL strains, respectively) (Fig. 3a-b; Additional file 2). Though falling short of our shared $>2 \log 2$ fold change threshold, insulinlike peptide gene, $d a f-28$, was the single most differentially regulated ins gene, exhibiting a polarised expression pattern that correlates inversely with both enhanced chemotaxis toward G. mellonella (S. carpocapsae Breton), and reduced nictation behaviour (S. carpocapsae UK1) (down-regulated 1.1 and $2.8 \log 2$ fold, $P<0.0001^{* * * *}$ in Breton relative to ALL and UK1, respectively; up-regulated 1.7 and $2.8 \log 2$ fold, $\mathrm{P}<0.0001^{\% * \%}$ in UK1 relative to ALL and Breton, respectively) (Fig. 2c-d; Additional file 2). Flp genes were comparatively less variant between strains, with $f l p-34$ exhibiting the largest pairwise expression change that correlates with increased chemotaxis behaviour in $S$. carpocapsae Breton (up-regulated 0.7 and $0.6 \log 2$ fold, $P<0.0001^{* * * * *}$, relative to UK1 and ALL strains, respectively) (Additional file 2). The tyrosine decarboxylase gene, $S c-t d c-1$, was the most differentially expressed neurotransmitter gene (down-regulated 1.4 and $1.9 \log 2$ fold, $\mathrm{P}<0.0001^{* * * * *}$ in $S$. 


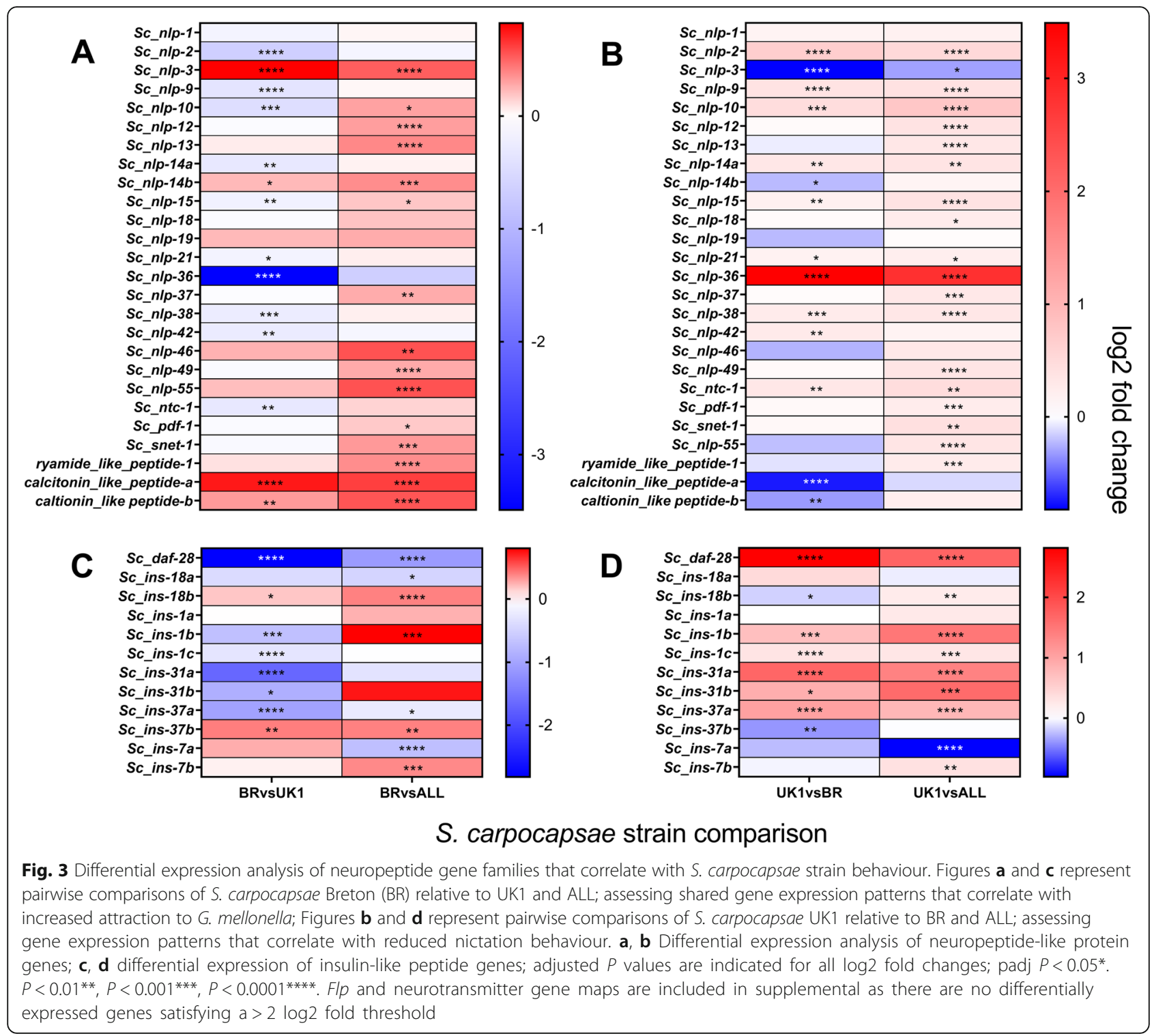

carpocapsae UK1 relative to ALL and Breton, respectively), correlating with decreased nictation behaviour, but falling short of a shared $>2 \log 2$ fold change threshold (Additional file 2).

\section{GPCR, innexin and ion channel genes}

Significant up-regulation of four srsx GPCR genes (Sc-srsx25v, Sc-srsx-3ii, Sc-srsx-22i, and Sc-srsx-24ii) (> $2 \log 2$ fold, $\left.P<0.0001^{* * * * *}\right)$ correlates with increased chemotaxis of $S$. carpocapsae Breton to the insect host G. mellonella, relative to UK1 and ALL strains (Fig. 4a; Additional file 2). By way of contrast, the Sc-srt-62 chemosensory GPCR gene was co-ordinately down-regulated in S. carpocapsae UK1, correlating with reduced nictation behaviour (down-regulated 2.6 and $2.5 \log 2$ fold, $P<0.0001^{\text {****** }}$ relative to ALL and Breton strains, respectively) (Fig. 4b; Additional file 2).
Although falling below our threshold of shared $>2 \log 2$ fold change across both strain pairwise comparisons, Scinx-7ii demonstrated the largest expression change in the innexin / gap junction gene family, correlating with increased chemotaxis behaviour (down-regulated 1.03 and $2.65 \log 2$ fold in S. carpocapsae Breton relative to UK1 and ALL strains, respectively; Fig. 4c-d; Additional file 2). Two paralogous asic-2 sodium channel genes were found to be differentially expressed, and inversely related to altered chemotaxis behaviour (Sc-asic-2ii; down-regulated 2.3 and $2.7 \log 2$ fold, $P<0.0001^{* * * *}$ in $S$. carpocapsae Breton, relative to ALL and UK1 strains, respectively; Fig. 4e, Additional file 2), and reduced nictation behaviour (Sc-asic-2i; up-regulated 2.9 and $3 \log 2$ fold, $P<0.0001^{* * * * *}$ in $S$. carpocapsae UK1, relative to ALL and Breton strains, respectively; Fig. 4f, Additional file 2). 


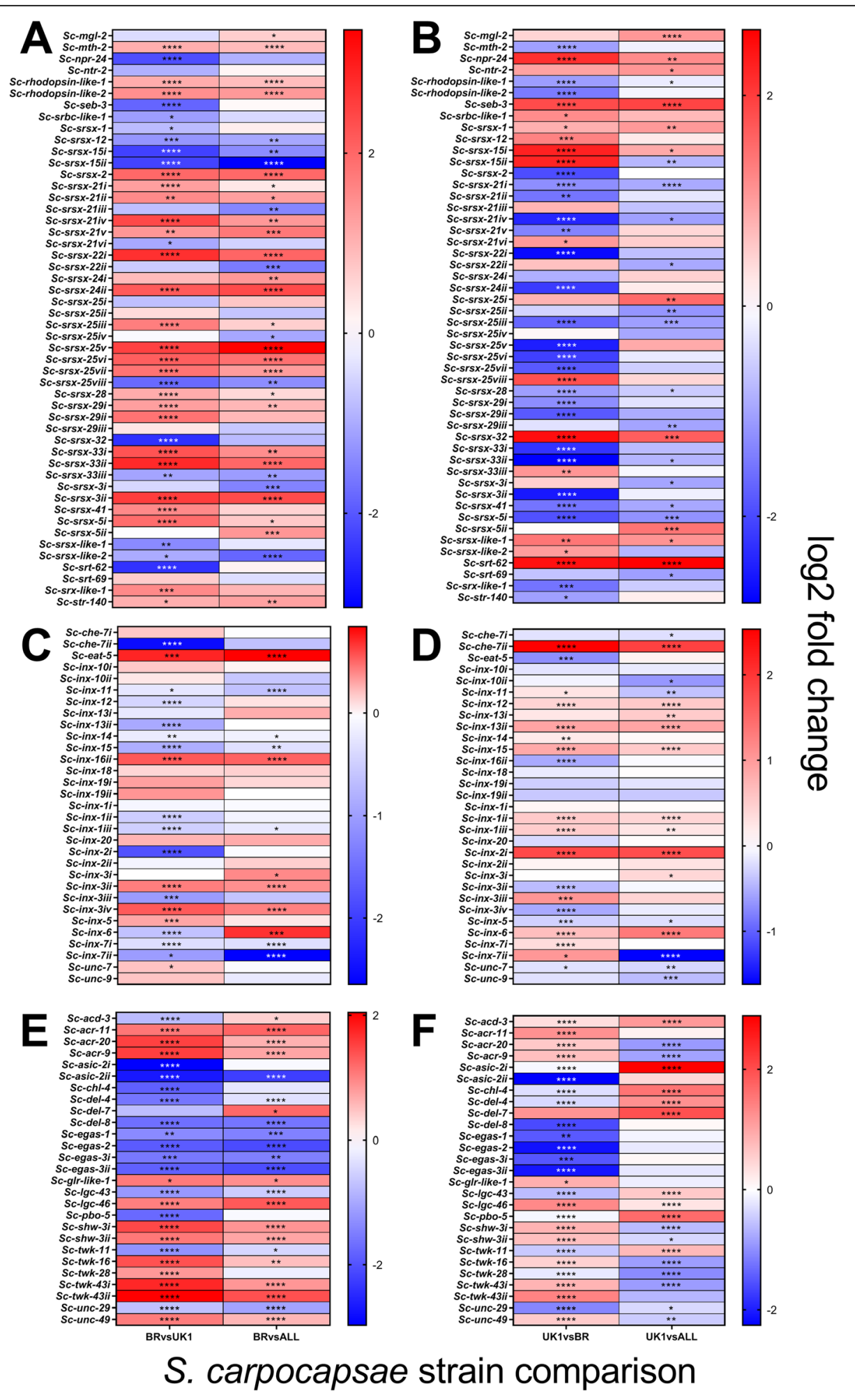

Fig. 4 (See legend on next page.) 
(See figure on previous page.)

Fig. 4 Differential expression analysis of GPCR, innexin and ion channel gene families that correlate with $\mathrm{S}$. carpocapsae strain behaviour. Figures a, $\mathbf{c}$ and $\mathbf{e}$ represent pairwise comparisons of $\mathrm{S}$. carpocapsae Breton (BR) relative to UK1 and All; assessing shared gene expression patterns that correlate with increased chemotaxis to G. mellonella; Figures $\mathbf{b}, \mathbf{d}$ and $\mathbf{f}$ represent pairwise comparisons of S. carpocapsae UK1 relative to BR and All; assessing gene expression patterns that correlate with reduced nictation behaviour. $\mathbf{a}$, $\mathbf{b}$ putative GPCR genes with $>1 \log 2$ fold difference between at least one of the pairwise comparisons; $\mathbf{c}$ and $\mathbf{d}$ putative innexin genes across all log2 fold changes; $\mathbf{e}$ and $\mathbf{f}$ putative ion channel genes with $>1$ log 2 fold difference between at least one of the pairwise comparisons. $P$ values are indicated for all log2 fold changes; padj $P<0.05^{*} . P<0.01^{* *}, P<0.001^{* *}, P<0.0001^{* * * *}$

\section{MicroRNA prediction and quantification}

Following miRDeep2 identification, quality control and manual curation, a total of 283 high confidence microRNA genes and 321 predicted mature microRNAs (306 of which are unique within the genome) were identified across a deep analysis ( $n=18$ libraries) of $S$. carpocapsae strains (Additional file 3). One hundred fifty of the unique mature microRNA sequences were previously identified in S. carpocapsae Breton [49], with an additional 156 unique mature microRNAs identified within this study. 102 (36\%) and 103 (36\%) S. carpocapsae Breton microRNAs were differentially expressed $\left(P<0.0001^{* * * * * *)}\right)$ relative to UK1 and ALL strains, respectively. 50 (17.6\%) S. carpocapsae UK1 microRNAs were differentially expressed $\left(P<0.0001^{* * * * * * *}\right)$ relative to $S$. carpocapsae ALL (Fig. 5; Additional file 3).
Three microRNAs (Sc-mir-117, Sc-mir-27, and Sc-mir-774) were highly differentially expressed, correlating with enhanced chemotaxis behaviour in S. carpocapsae Breton ( $>6$ $\log 2$ fold, $P<0.0001^{* * * *}$ relative to UK1 and All strains). A further 18 microRNAs were likewise differentially expressed and correlated with enhanced chemotaxis behaviour $(>2$ $\log 2$ fold, $P<0.0001^{* * * \%}$ ) (Fig. 6a; Additional files 3 and 4). Comparatively fewer microRNAs were differentially regulated and correlated with reduced nictation behaviour in $S$. carpocapsae UK1, with Sc-mir-772 (up-regulated 12.9 and $12.7 \log 2$ fold, $P<0.0001^{*} *$ relative to Breton and All strains, respectively) and Sc-mir-773 (up-regulated 8.7 and $8.5 \log 2$ fold, $P<0.0001^{* * * * *}$ relative to Breton and All strains, respectively) representing notable exceptions. A further five microRNAs (Sc-mir-754, Sc-mir-756, Sc-mir-760, Sc-let-7

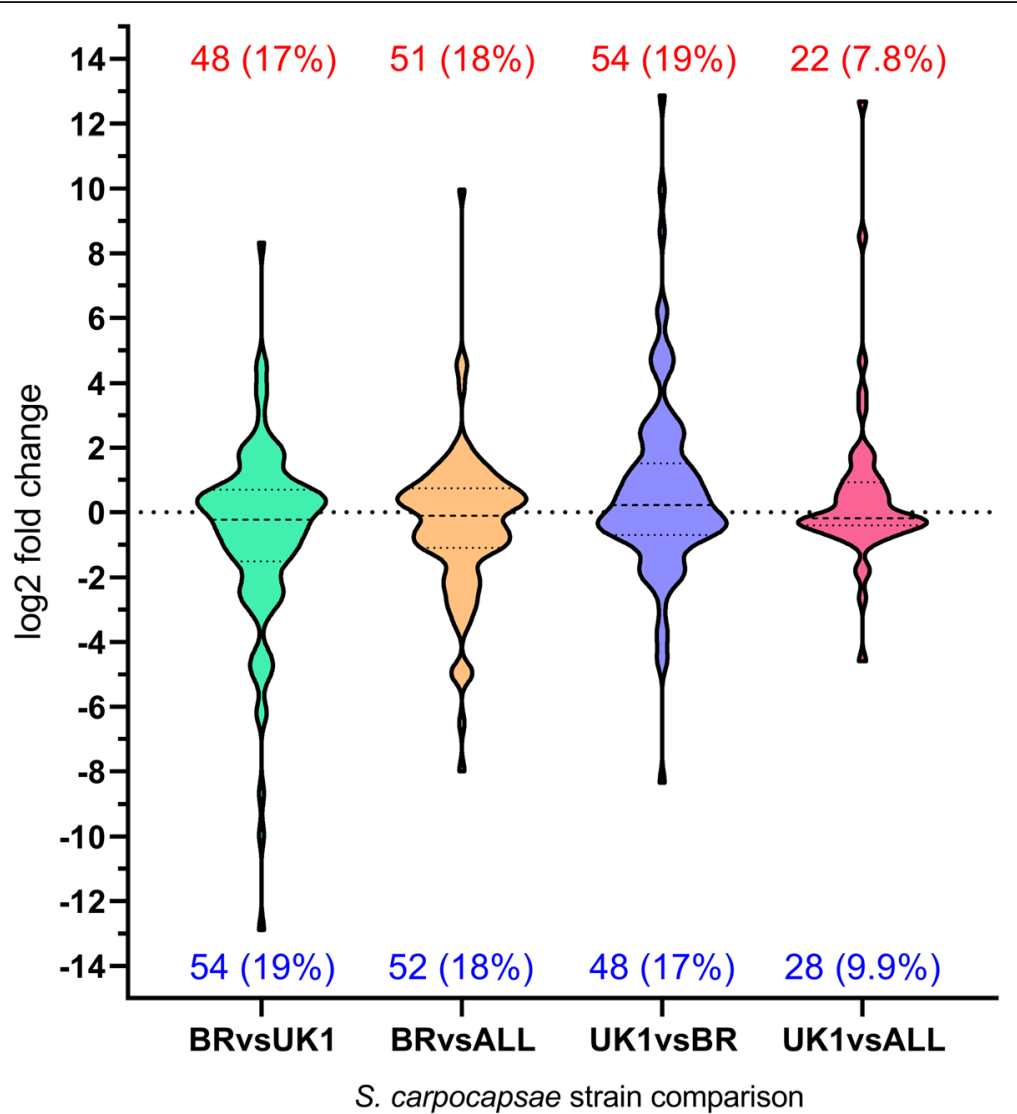

Fig. 5 Violin plot showing significantly up-regulated and down-regulated microRNA genes across pairwise strain comparisons. Statistically significant $\left(P<0.0001^{* * * *}\right)$ differentially expressed genes are plotted irrespective of log2 fold change. Total number and relative percentage of up-regulated and down-regulated microRNA genes are presented above and below each plot, respectively 
A

\begin{tabular}{|c|c|c|}
\hline \multirow{5}{*}{$\begin{array}{l}\text { Sc-miR-108- } \\
\text { Sc-miR-117 } \\
\text { Sc-miR-138 } \\
\text { Sc-miR-147 } \\
\text { Sc-miR-151- }\end{array}$} & $* * * *$ & $* * * *$ \\
\hline & $\star \star \star \star \star *$ & $* * * *$ \\
\hline & $* * * *$ & $* * * *$ \\
\hline & $* * * *$ & $* * * *$ \\
\hline & $* * * *$ & $* * * *$ \\
\hline \multirow{7}{*}{$\begin{array}{r}\text { Sc-miR-156 } \\
\text { Sc-miR-161 } \\
\text { Sc-miR-176 } \\
\text { Sc-miR-184 } \\
\text { Sc-miR-190 } \\
\text { Sc-miR-211 } \\
\text { Sc-miR-27 }\end{array}$} & $* * * *$ & $* * * *$ \\
\hline & $* * * *$ & 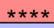 \\
\hline & $* * * *$ & $* * * *$ \\
\hline & $* * * *$ & $* * * *$ \\
\hline & $* * *$ & $* * * *$ \\
\hline & $\star \star \star * *$ & $* * * *$ \\
\hline & $* * * *$ & $* * * *$ \\
\hline \multirow{6}{*}{$\begin{array}{l}\text { Sc-miR-283 } \\
\text { Sc-miR-299 } \\
\text { Sc-miR-301 } \\
\text { Sc-miR-306- } \\
\text { Sc-miR-316 - } \\
\text { Sc-miR-337 }\end{array}$} & $* * * *$ & $* * * *$ \\
\hline & $\star \star * \star *$ & $* * * *$ \\
\hline & $* * * *$ & $* * * *$ \\
\hline & $* * * *$ & $* * * *$ \\
\hline & $* * * *$ & $* * * *$ \\
\hline & $* * * *$ & $* * * *$ \\
\hline \multirow{3}{*}{$\begin{array}{l}\text { Sc-miR-338 } \\
\text { Sc-miR-351 } \\
\text { Sc-miR-754 }\end{array}$} & $* * * *$ & $* * * *$ \\
\hline & $* * * *$ & $* * * *$ \\
\hline & $* * * *$ & $* * * *$ \\
\hline \multirow{2}{*}{$\begin{array}{l}\text { Sc-miR-756 - } \\
\text { Sc-miR-757 }\end{array}$} & $* * * *$ & $* * * *$ \\
\hline & $* * * *$ & $* *$ \\
\hline \multirow{3}{*}{$\begin{array}{l}\text { Sc-miR-759- } \\
\text { Sc-miR-760- } \\
\text { Sc-miR-761- }\end{array}$} & $* * * *$ & $* *$ \\
\hline & $* * * *$ & $\star * * *$ \\
\hline & $* * * *$ & \\
\hline \multirow{2}{*}{$\begin{array}{r}\text { Sc-miR-762- } \\
\text { Sc-let-7-5p- }\end{array}$} & $* * * *$ & $* * * *$ \\
\hline & $* * * *$ & \\
\hline \multirow{4}{*}{$\begin{array}{l}\text { Sc-miR-772 } \\
\text { Sc-miR-773 } \\
\text { Sc-miR-774 } \\
\text { Sc-miR-775 }\end{array}$} & $* * * *$ & \\
\hline & $* * * *$ & \\
\hline & $* * * *$ & $* * * *$ \\
\hline & $* * * *$ & $* * * *$ \\
\hline \multirow{2}{*}{$\begin{array}{r}\text { Sc-miR-84-5pi } \\
\text { Sc-miR-91 }\end{array}$} & $* * * *$ & $* * * *$ \\
\hline & $\star * * *$ & $* * * *$ \\
\hline
\end{tabular}

B

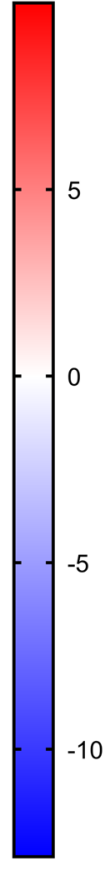

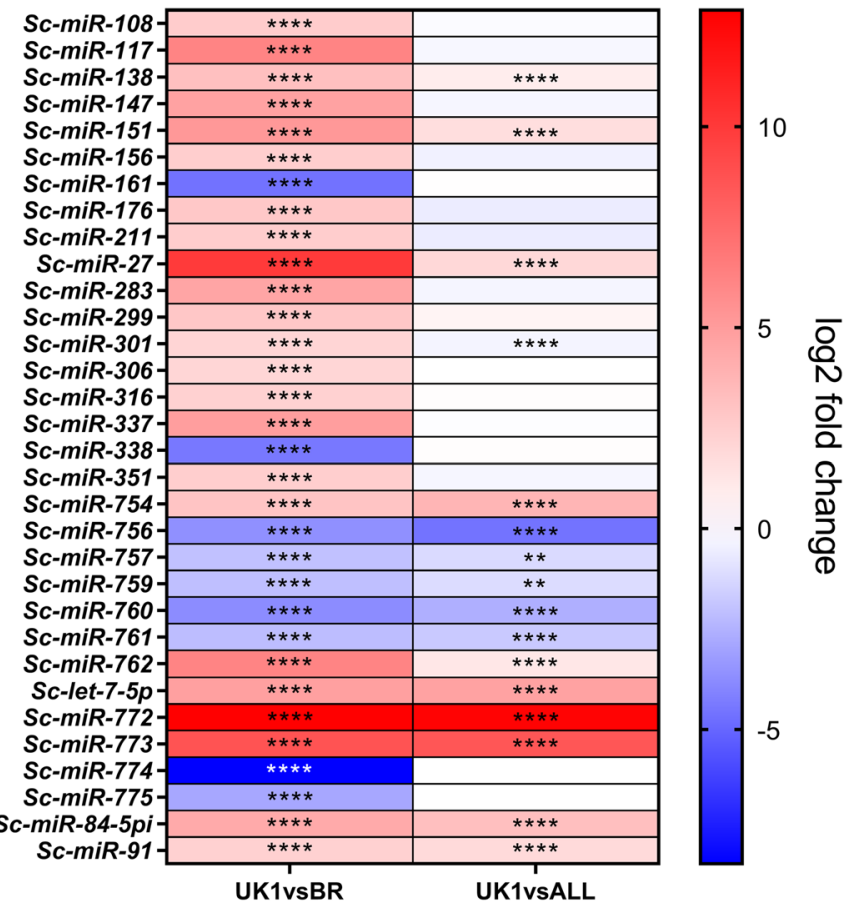

\section{S. carpocapsae strain comparison}

Fig. 6 Differential expression analysis of microRNAs that correlate with S. carpocapsae strain behaviour. Heatmaps showing differential expression of microRNAs with at least one pairwise expression difference of $>2 \log 2$ fold change, $P<0.0001^{* * * *}$. a Differentially expressed microRNAs in $S$. carpocapsae Breton, relative to ALL and UK1 strains; assessing shared expression patterns that correlate with increased chemotaxis behaviour. $\mathbf{b}$ Differentially expressed microRNAs in S. carpocapsae UK1, relative to ALL and Breton strains; assessing shared expression patterns that correlate with reduced nictation behaviour (Additional file 4)

and Sc-mir-84-5pi) were likewise differentially expressed and correlated with reduced nictation behaviour ( $>2 \log 2$ fold, $P<0.0001^{* * * * *)}$ ) (Fig. 6b; Additional files 3 and 4).

\section{MicroRNA target analysis}

In silico microRNA target prediction through miRanda suggests a substantial bias towards interactions with gene 5'UTRs. A total of 231,120 microRNA interactions, representing 247,237 binding sites are predicted for the 5'UTR of $S$. carpocapsae genes, relative to 127,796 microRNA interactions, representing 132,309 binding site predictions within the 3'UTR of S. carpocapsae genes. Through screening each of the most differentially expressed $(>2 \log 2$ fold change, $P<0.0001 \% * \%)$ microRNAs that correlate with behavioural variants across pairwise comparisons (Fig. 6; Additional file 4), we identify a substantial number of predicted interactions with neuronal gene families considered in this study (Table 1). These datasets demonstrate potential cooperation between microRNAs that are predicted to interact with shared target genes. For example, the ion channel gene Sc-asic-2ii is a predicted target for Sc-mir147, Sc-mir-301-3p, and Sc-mir-316, all highly differentially expressed, and correlated with increased chemotaxis behaviour (Table 1; Fig. 5a). Similarly, the let-7 family members, Sc-mir-84-5pi and Sc-let-7 co-ordinately target two ins-1 paralogues; both microRNAs are highly differentially expressed, and correlated with reduced nictation behaviour in S. carpocapsae UK1 (Fig. 5b). Our data also suggest that different microRNAs are predicted to interact with and converge on a number of shared neuronal gene targets considered here (Table 1). For example, Sc-mir$301-3 p$ is predicted to simultaneously target the $t w k-12$, egas- 1 and asic- 2 ion channel genes, alongside the $n l p-39$ neuropeptide-like protein gene, correlating with increased chemotaxis behaviour (Table 1; Figs. 5a and 1a). MiRanda microRNA target predictions across both strict and default settings for global $5^{\prime}$ and 3'UTRs are presented in Additional files 5, 6, 7 and 8 .

\section{Differential isoform usage between strains}

Two genes implicated in the synthesis and transport of classical neurotransmitters were found to exhibit statistically significant differences in isoform preference across $S$. carpocapsae strains following RSEM analysis (Figs. 6, 7 and 8; Additional file 9). Interestingly, these isoform variants exhibit altered UTR sequences in 
Table 1 Predicted gene targets for differentially expressed microRNAs ( $>2 \log 2$ fold, $P<0.0001)$ that correlate with $S$. carpocapsae strain behaviour. Differentially expressed S. carpocapsae microRNAs were assessed for binding sites across $5^{\prime}$ and $3^{\prime} U T R s$ of all ion channel, innexin, GPCR, neurotransmitter and neuropeptide genes. Ce target refers to direct 1 to 1 gene orthologues that are biochemically confirmed microRNA targets in C. elegans [20]. U refers to the number of predicted interacting microRNAs following default microRNA target prediction; $S$ refers to the number of predicted interacting microRNAs following strict microRNA target prediction. Within the implicated behaviour column, "Both (inverse int.)" refers to involvement in both behaviours, through polarised regulation states (significantly upregulated for one, and significantly downregulated for the other)

\begin{tabular}{|c|c|c|c|c|c|c|c|c|}
\hline & microRNA & Gene & Gene Name & Gene family & Ce target & D & $S$ & Implicated behaviour \\
\hline \multirow[t]{8}{*}{$\overline{3^{\prime} U T R}$} & sc-miR-301-3p & L596_g12890.t2 & twk-12 & Ion Channel & y & 2 & 1 & Chemotaxis \\
\hline & sc-miR-301-3p & L596_g12890.t3 & twk-12 & Ion Channel & Y & 2 & 1 & Chemotaxis \\
\hline & sc-miR-301-3p & L596_g12890.t1 & twk-12 & Ion Channel & y & 2 & 1 & Chemotaxis \\
\hline & sc-miR-759 & L596_g2695.t1 & acr-21 & Ion Channel & & 2 & 1 & Nictation \\
\hline & sc-miR-138 & L596_g3511.t1 & eat-5 & Innexin & & 2 & 0 & Chemotaxis \\
\hline & sc-miR-117 & L596_g24535.t1 & ador-1 & GPCR & & 2 & 0 & Chemotaxis \\
\hline & sc-miR-283 & L596_g24535.t1 & ador-1 & GPCR & & 2 & 0 & Chemotaxis \\
\hline & sc-miR-84-5pi & L596_g1430.t1 & ins- 16 & Neuropeptide & y & 2 & 1 & Nictation \\
\hline \multirow[t]{31}{*}{$5^{\prime} \mathrm{UTR}$} & sc-miR-147 & L596_g23064.t1 & asic-2ii & Ion Channel & & 2 & 2 & Chemotaxis \\
\hline & sc-miR-147 & L596_g14933.t1 & unc-36 & Ion Channel & & 2 & 2 & Chemotaxis \\
\hline & sc-miR-156 & L596_g11170.t1 & egas-1 & Ion Channel & & 2 & 2 & Chemotaxis \\
\hline & sc-miR-176 & L596_g11280.t1 & $\operatorname{lgc}-22$ & Ion Channel & & 2 & 2 & Chemotaxis \\
\hline & sc-miR-184 & L596_g11170.t1 & egas-1 & Ion Channel & & 3 & 0 & Chemotaxis \\
\hline & sc-miR-184 & L596_g13756.t1 & acr-25 & Ion Channel & & 2 & 2 & Chemotaxis \\
\hline & sc-miR-190 & L596_g21438.t2 & unc-2 & Ion Channel & y & 3 & 3 & Chemotaxis \\
\hline & sc-miR-190 & L596_g21438.t1 & unc-2 & Ion Channel & y & 3 & 3 & Chemotaxis \\
\hline & sc-miR-190 & L596_g25880.t2 & slo-1 & Ion Channel & y & 2 & 2 & Chemotaxis \\
\hline & sc-miR-190 & L596_g25880.t1 & slo-1 & Ion Channel & y & 2 & 2 & Chemotaxis \\
\hline & sc-miR-211 & L596_g19116.t1 & unc- 49 & Ion Channel & y & 5 & 5 & Chemotaxis \\
\hline & sc-miR-299 & L596_g11170.t1 & egas-1 & Ion Channel & & 2 & 1 & Chemotaxis \\
\hline & $s c-m i R-301-3 p$ & L596_g11170.t1 & egas-1 & Ion Channel & & 3 & 1 & Chemotaxis \\
\hline & sc-miR-301-3p & L596_g23064.t1 & asic-2ii & Ion Channel & & 2 & 1 & Chemotaxis \\
\hline & sc-miR-301-5pi & L596_g9175.t1 & clhm-1 & Ion Channel & & 2 & 2 & Chemotaxis \\
\hline & sc-miR-301-5pi & L596_g13756.t1 & $a c r-25$ & Ion Channel & & 2 & 1 & Chemotaxis \\
\hline & sc-miR-301-5pii & L596_g13756.t1 & acr- -25 & Ion Channel & & 2 & 1 & Chemotaxis \\
\hline & sc-miR-301-5pii & L596_g11170.t1 & egas-1 & Ion Channel & & 2 & 2 & Chemotaxis \\
\hline & sc-miR-301-5pii & L596_g9175.t1 & clhm-1 & Ion Channel & & 2 & 0 & Chemotaxis \\
\hline & sc-miR-306 & L596_g21215.t1 & twk-46 & Ion Channel & & 2 & 1 & Chemotaxis \\
\hline & sc-miR-316 & L596_g14933.t1 & unc-36 & Ion Channel & & 2 & 2 & Chemotaxis \\
\hline & sc-miR-316 & L596_g23064.t1 & asic-2ii & Ion Channel & & 2 & 2 & Chemotaxis \\
\hline & sc-miR-337 & L596_g9175.t1 & clhm-1 & Ion Channel & & 2 & 0 & Chemotaxis \\
\hline & sc-miR-338 & L596_g14875.t1 & $k v s-5$ & Ion Channel & & 2 & 1 & Chemotaxis \\
\hline & sc-miR-351 & L596_g15440.t1 & twk-43 & Ion Channel & & 2 & 1 & Chemotaxis \\
\hline & sc-miR-756 & L596_g9186.t2 & twk-2 & Ion Channel & & 2 & 2 & Nictation \\
\hline & sc-miR-756 & L596_g9186.t1 & twk-2 & Ion Channel & & 2 & 2 & Nictation \\
\hline & sc-miR-759 & L596_g15570.t1 & shk-1 & Ion Channel & y & 2 & 1 & Nictation \\
\hline & sc-miR-772 & L596_g15357.t1 & clh-5 & Ion Channel & & 2 & 1 & Nictation \\
\hline & sc-miR-772 & L596_g15357.t2 & clh-5 & Ion Channel & & 2 & 1 & Nictation \\
\hline & sc-miR-772 & L596_g11576.t1 & $a v r-14$ & Ion Channel & y & 2 & 0 & Nictation \\
\hline
\end{tabular}


Table 1 Predicted gene targets for differentially expressed microRNAs ( $>2 \log 2$ fold, $P<0.0001$ ) that correlate with $S$. carpocapsae strain behaviour. Differentially expressed $S$. carpocapsae microRNAs were assessed for binding sites across $5^{\prime}$ and $3^{\prime} U T R s$ of all ion channel, innexin, GPCR, neurotransmitter and neuropeptide genes. Ce target refers to direct 1 to 1 gene orthologues that are biochemically confirmed microRNA targets in C. elegans [20]. U refers to the number of predicted interacting microRNAs following default microRNA target prediction; $\mathrm{S}$ refers to the number of predicted interacting microRNAs following strict microRNA target prediction. Within the implicated behaviour column, "Both (inverse int.)" refers to involvement in both behaviours, through polarised regulation states (significantly upregulated for one, and significantly downregulated for the other) (Continued)

\begin{tabular}{|c|c|c|c|c|c|c|c|}
\hline microRNA & Gene & Gene Name & Gene family & Ce target & $\mathrm{D}$ & $S$ & Implicated behaviour \\
\hline sc-miR-775 & L596_g20944.t1 & twk-29 & Ion Channel & & 2 & 2 & Chemotaxis \\
\hline sc-miR-91 & L596_g12530.t1 & unc-58 & Ion Channel & & 2 & 1 & Nictation \\
\hline sc-miR-759 & L596_g25584.t1 & unc-7 & Innexin & Y & 2 & 2 & Nictation \\
\hline sc-miR-138 & L596_g9922.t1 & gar-2 & GPCR & y & 2 & 0 & Chemotaxis \\
\hline sc-miR-151 & L596_g14843.t1 & $n p r-26$ & GPCR & & 2 & 2 & Both (inverse int.) \\
\hline sc-miR-184 & L596_g17321.t1 & $n p r-23$ & GPCR & y & 2 & 0 & Chemotaxis \\
\hline sc-miR-27 & L596_g22293.t1 & C30A5.10 & GPCR & & 2 & 1 & Both (inverse int.) \\
\hline sc-miR-301-5pi & L596_g14261.t2 & $n p r-11$ & GPCR & & 2 & 1 & Chemotaxis \\
\hline sc-miR-351 & L596_g16439.t1 & $\operatorname{srs} x-24$ & GPCR & & 2 & 2 & Chemotaxis \\
\hline sc-miR-756 & L596_g17321.t1 & $n p r-23$ & GPCR & y & 2 & 1 & Nictation \\
\hline sc-miR-761 & L596_g25676.t1 & gar-1 & GPCR & & 2 & 1 & Nictation \\
\hline sc-miR-773 & L596_g25676.t1 & gar-1 & GPCR & & 2 & 0 & Nictation \\
\hline Sc-let-7 & L596_g13647.t1 & ins-1a & Neuropeptide & y & 2 & 1 & Nictation \\
\hline$s c-m i R-301-3 p$ & L596_g14298.t1 & $n / p-49$ & Neuropeptide & & 2 & 1 & Chemotaxis \\
\hline sc-miR-84-5pi & L596_g5500.t2 & vglu-2 & Neurotransmission & & 2 & 2 & Both (inverse int.) \\
\hline
\end{tabular}

additional to altered exon usage. In silico microRNA target predictions were conducted for all predicted microRNAs relative to the respective isoform UTRs, identifying quantitative differences in predicted microRNA interactions with the various gene isoforms (Table 2; Additional file 9).

\section{Discussion}

This study has generated high quality transcriptomes $(n=18)$ and small RNA libraries $(n=18)$ for the IJ stage of three S. carpocapsae strains, and has catalogued a number of protein-coding and non-coding RNAs that are differentially expressed between strains with distinct hostfinding behaviours. We have applied an arbitrary threshold of $>2 \log 2$ fold change $\left(P<0.0001^{* * * * *}\right)$ across both pairwise strain comparisons in order to link gene expression markers to behavioural differences. Many of these genes have already been shown to regulate behaviour in the model nematode C. elegans, however none have been implicated specifically in host-finding behaviours of parasitic nematodes. These data support the potential benefit of establishing and investing in natural diversity resources for parasitic nematodes, alongside more expansive studies in the model nematode $C$. elegans $[50,51]$.

GO term enrichment revealed that a range of gene families and biological processes were enriched in differentially expressed gene sets that correspond with behavioural variation in $S$. carpocapsae strains (Additional file 1).
Transmembrane transport was the single most enriched biological process when considering either nictation or chemotaxis behaviours, following by a series of metabolic and biosynthetic processes. Of particular interest, several neuronal functions were also enriched. Given that we know much of how neuronal genes regulate behaviour in the model nematode $C$. elegans, neuronal gene families became the focus of our analysis. Only one neuropeptide gene, $n l p$ 36 , was found to exhibit differential expression ( $>2 \log 2-$ fold, $P<0.0001^{* * * * *)}$ that correlates with distinct strain behaviours. NLP-36 is positively regulated by the cyclic nucleotide-gated channel subunit TAX-2 in C. elegans, which is implicated in the regulation of olfaction, chemosensation, thermosensation and axon guidance for a number of sensory neurons $[52,53]$. In S. carpocapsae UK1, significant up-regulation of $n l p-36$ correlates with reduced nictation behaviour (Figs. $1 \mathrm{~b}$ and $3 \mathrm{~b}$ ). Down-regulation of insulin signalling during $C$. elegans development has been found to influence nictation behaviour in dauer juveniles, indicating a tuning of behaviour to environmental conditions [10]. Gruner et al. [54] point to a potential neuronal programming circuit for behavioural adjustment in C. elegans through the combined influence of insulin signalling, TRPV signalling and agonism of NPR-1, a known receptor of neuropeptides, FLP-21 and FLP-18. Although the differential expression of daf-28 does not meet the shared $>2$ $\log 2$ fold change threshold we have applied to sequencing datasets, it is nonetheless down-regulated in S. carpocapsae 

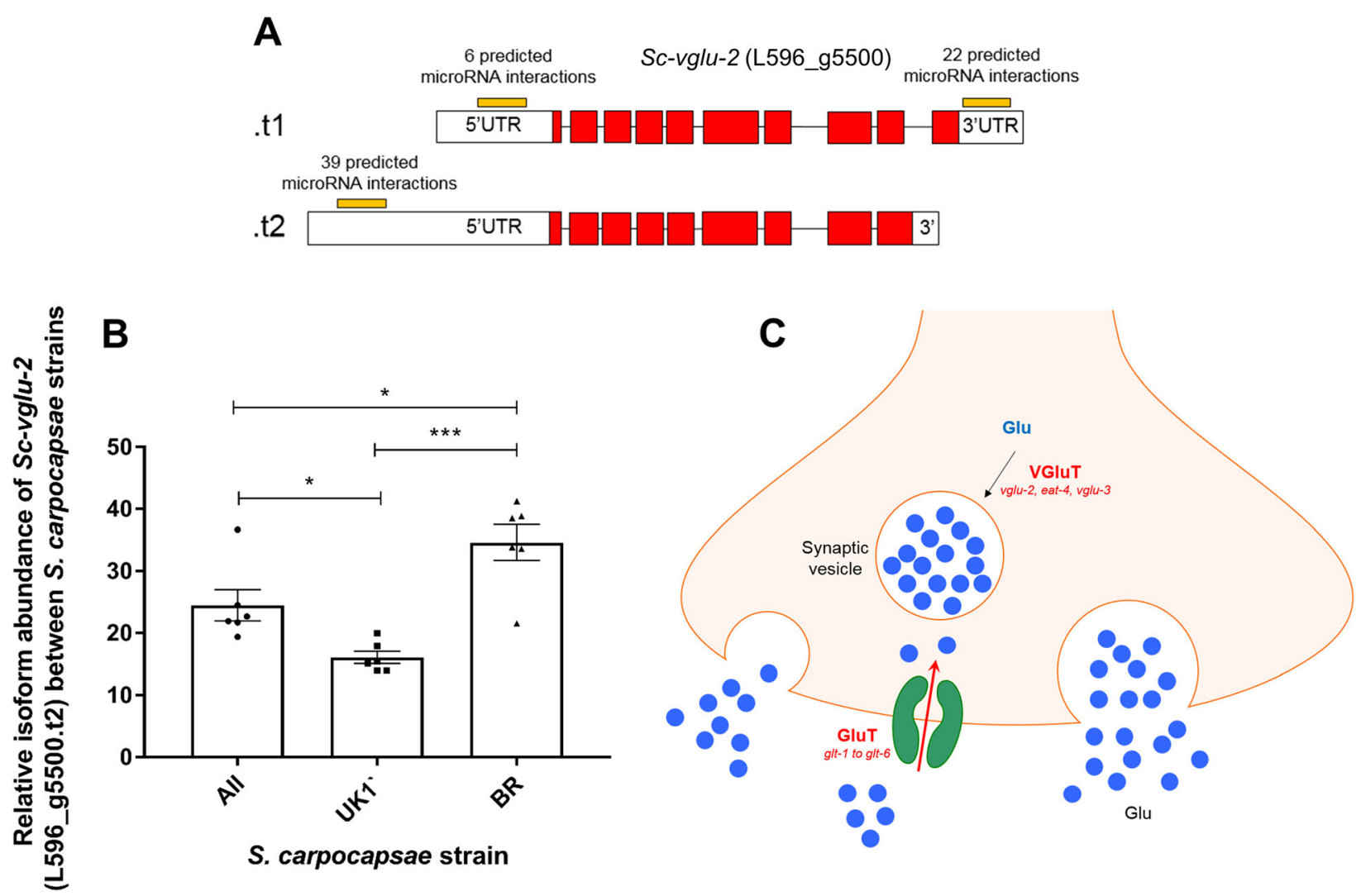

Fig. 7 Variation in isoform preference may alter microRNA targeting of the vesicular glutamine transporter gene Sc-vglu-2 between strains of S. carpocapsae. a Diagrammatic depiction of isoform structure between predicted variants (not to scale); white boxes indicate UTRs, red boxes indicate exons. $\mathbf{b}$ Graph indicating relative percentage abundance of L596_g5500.t2 across S. carpocapsae strains ( $n=6$ independent libraries per strain). One way ANOVA and Tukey's multiple comparison tests were conducted using Graphpad Prism 7.02. $P<0.05^{*}, P<0.001^{* *}$. c Diagrammatic depiction of the relative position of VGluT in a glutamatergic neuron

Breton, relative to the other strains, correlating with enhanced chemotaxis toward G. mellonella. Conversely, daf28 was up-regulated in UK1 relative to both Breton and All strains (Fig. 3a, b), correlating with reduced nictation (Fig. 1b). As was found for the two asic-2 sodium channel paralogues, daf-28 and other genes could function as part of a neurobiological switch, tuning behavioural strategy toward either migratory or stationary host-finding modes. It may be informative to track the dynamic abundance of such genes in IJs naturally enacting and transitioning between these behaviours, or in conditions that are known to enhance or suppress these behaviours to further strengthen the correlation of expression with behaviour $[55,56]$.

The up-regulation of srsx GPCR genes in S. carpocapsae Breton correlates with enhanced chemotaxis to the lab host insect G. mellonella (Fig. 4a). One of these genes is orthologous to a cluster of srsx genes (srsx -22 and srs $x-24)$ that are enriched in dauer stage C. elegans. It has been shown that dauer stage Caenorhabditis spp. are attracted to certain insect species, increasing the opportunity to engage in phoresis [57]. This cluster of shared srsx GPCRs may therefore mediate this attraction, in isolation, or in synergy with other such receptors. Two asic-2 sodium channel paralogues (denoted here as $S c$-asic- $2 i$ and $S c$ asic-2ii) are also notable as being differentially expressed and inversely correlated with both increased chemotaxis and reduced nictation behaviours (Fig. 4e-f). ASIC-2 is known to regulate aspects of nematode body posture and mechanosensation in C. elegans [58].

Small non-coding RNAs, including microRNAs, are increasingly implicated in complex aspects of biology and behaviour [51, 59-61]. We conducted a deep analysis of small RNA profiles across $S$. carpocapsae strains, and found a substantial degree of variation in relative abundance (Figs. 5 and 6, Additional file 3). Numerous microRNAs are differentially expressed, and correlate with behavioural differences across pairwise comparisons (Fig. 6, Additional file 4). Key to extrapolating biologically relevant information from microRNA networks is the identification of gene targets. Although there are many in silico tools that predict microRNA-mRNA transcript interactions, false positives are likely to be common [62]. Whilst many factors influence the reality and significance of predicted interactions, bioavailability of target 


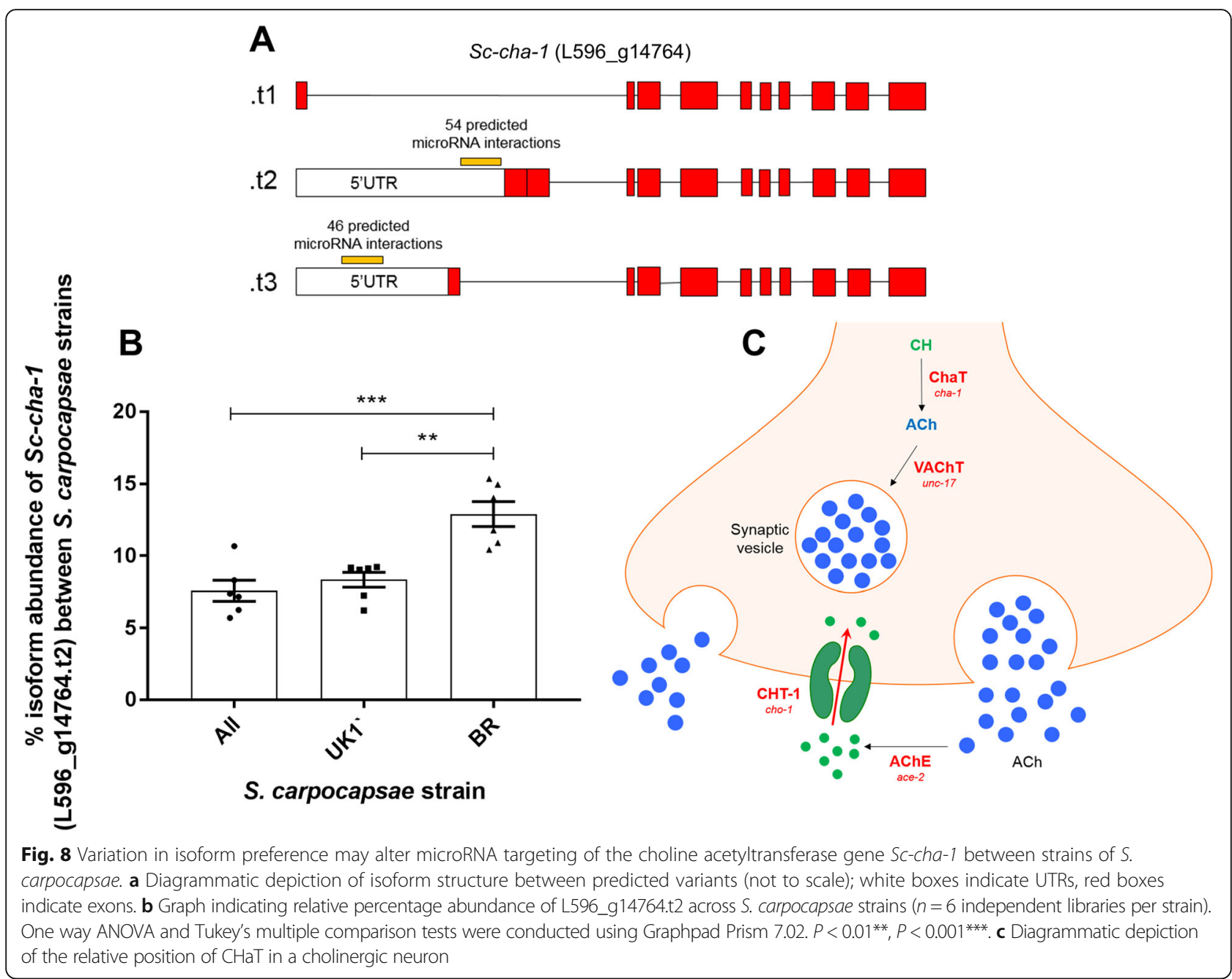

gene and microRNA in terms of spatial and temporal expression patterns will be key, along with the number of available microRNA binding sites, the relative enthalpy of binding interactions, and local competition for available microRNAs. In order to build on the basic knowledge presented in this study, it will be necessary to biochemically validate gene transcripts as microRNA targets through Argonaute ClIP-seq [45], and further, to demonstrate colocalisation of microRNA and target mRNA transcript to confirm interaction of discrete partners. This represents a substantial, but necessary task if we are to unravel the biological significance of microRNA regulation in the context of complex phenotypes and behaviours. Previous publications have employed a hierarchical and cooperative in silico target prediction approach using several programs simultaneously to arrive at an agreed set of targets [59]. Whilst this will certainly reduce the complexity of any target gene set, it will also constrain the output according

Table 2 Differential microRNA targeting across Sc-cha-1 and Sc-vglu-2 gene isoform 5' and 3'UTRs. D denotes number of predicted microRNA interactions following default target prediction in miRanda; $\mathbf{S}$ denotes number of predicted microRNA interactions following strict target prediction in miRanda. Numbers in brackets indicate the relative number of binding sites across both D and $\mathrm{S}$ settings

\begin{tabular}{|c|c|c|c|c|c|c|c|}
\hline \multirow[t]{2}{*}{ Transcript ID } & \multicolumn{3}{|c|}{ Mean \%lsoform abundance } & \multicolumn{2}{|c|}{$N^{\circ}$ microRNA interactions in $3^{\prime} U T R$ (binding sites) } & \multicolumn{2}{|c|}{$\mathrm{N}^{\circ}$ microRNA interactions in $5^{\prime} U T R$ (binding sites) } \\
\hline & $\overline{\mathrm{ALL}}$ & UK1 & $\mathrm{BR}$ & $\bar{D}$ & $\mathrm{~S}$ & $\bar{D}$ & $\mathrm{~S}$ \\
\hline L596_g14764.t1 & $90.9 \%$ & $90.1 \%$ & $85.8 \%$ & - & - & - & - \\
\hline L596_g14764.t2 & $7.6 \%$ & $8.3 \%$ & $12.9 \%$ & - & - & $54(57)$ & $36(36)$ \\
\hline L596_g14764.t3 & $1.5 \%$ & $1.6 \%$ & $1.3 \%$ & - & - & $46(49)$ & $31(31)$ \\
\hline L596_g5500.t1 & $75.5 \%$ & $83.9 \%$ & $65.4 \%$ & $22(22)$ & $5(5)$ & 6 & $4(4)$ \\
\hline L596_g5500.t2 & $24.5 \%$ & $16.1 \%$ & $34.6 \%$ & - & - & $39(49)$ & $25(27)$ \\
\hline
\end{tabular}


to the most stringent program, leading inevitably to false negatives when perfect seed site complementarity is required by one or more programs. Here we have employed a dual analysis strategy within the miRanda discovery environment [43], using both default and strict discovery modes; the latter requires perfect seed site complementarity between microRNA and target mRNA transcript. Collectively, this strategy maps well to biochemicallyvalidated microRNA target interactions, including those that do not require perfect seed site binding [45]. However, as with any in silico prediction approach, biological validation is still required to corroborate interactions.

Our datasets suggest a strong microRNA target site enrichment within predicted 5'UTRs of S. carpocapsae. This may be biologically significant, or could represent an artefact of computational UTR prediction. Ultimately, higher confidence interactions could be established by sequencing full length transcripts and confirming UTR identity on a transcriptome wide scale. In silico target prediction for differentially expressed ( $>2 \log 2$ fold, $P<0.0001^{* * * *}$ ) and behaviourally correlated microRNAs points to instances of potential microRNA co-operation. For example, the ion channel genes Sc-asic-2ii and Sc-egas-1 are predicted targets for three and five individual differentially expressed microRNAs each (Table 1). The neuropeptide GPCR gene, Sc-npr-23 is likewise targeted independently by two differentially expressed microRNAs. The differential expression pattern of each targeting microRNA correlates with altered chemotaxis behaviour, suggesting that microRNAs may also drive behavioural variation (Table 1).

The predicted targeting of Sc-npr-11 by sc-miR-301-5pi reveals that UTR sequence variation between the two annotated npr-11 isoforms allows the dominant isoform (representing 75\% of all $n p r-11$ transcript across strains) to escape mir-301-5pi interaction (Table 1; Additional file 9). Likewise, variation in the 5'UTR and 3'UTRs of Sc-vglu-2 and Sc-cha-1 (Figs. 6 and 7, Table 2) reveal a potential quantitative difference in microRNA interaction events across different gene isoforms. In the case of Sc-cha-1, gene-level regulation of microRNA target site visibility could represent a strain specific mechanism to dampen global cholinergic signalling, or could allow selective dampening in a subset of cholinergic neurons. Ascertaining the relevance of isoform variance as it relates to microRNA interactions will require detailed gene localisation and functional studies. Our datasets highlight $5^{\prime}$ and $3^{\prime}$ UTR variation as a factor in differential microRNA target site visibility, although it seems especially likely that alternative polyadenylation signals in the 3'UTR of protein-coding genes will exert substantial control over microRNA target site visibility, especially given the evident pervasiveness of 3'UTR variation in nematodes [63, 64]. Any such regulation could modify the relative percentage of gene isoform variants accessible to microRNAs, which could allow for cell, or tissue-specific regulatory events that may be difficult to ascertain from organism-wide datasets. Collectively, our data point to a complex co-regulatory environment involving gene-specific isoform variation, and microRNA transcriptional regulation that is likely to influence various aspects of biology, including behaviour.

In the same way that "the best model for a cat is several cats" [65], the best model for a nematode parasite of vertebrates, is a sustainable population of the very same. However, this might not always be preferable in terms of ethics, available tools, sustainability or reproducibility. 'Model' status for any organism is contingent on high quality genomic and transcriptomic resources, easily amenable research tools, both in terms of genetic and molecular manipulation, alongside robust behavioural and phenotypic assays. Ease of culture, handling, and short generation times should also be major considerations. A model organism and the data generated from it must also be sufficiently relevant to trigger near-term impact on other species that have implications for health and economy. The datasets presented in this study build upon a growing catalogue of tools and resources for Steinernema spp. entomopathogenic nematodes $[9,21,30,49,65-67]$. The close phylogenetic relationship between Steinernema spp. and economically important mammalian parasites [68], coupled with striking behavioural similarities, and a pathogenic life style that can be fully recapitulated in a Petri dish suggests that these entomopathogenic nematodes could represent attractive and convenient new surrogate models for parasite biology and behaviour. In addition to their potential worth as model organisms, Steinernema spp. have attracted considerable attention as bioinsecticidal agents [69]. The interest in Steinernema spp. as biological models, and as economically relevant end-point organisms in their own right represents a unique proposition for researchers interested in comparative biology, behaviour and host-parasite interactions.

\section{Conclusions}

This study is the first to identify differentially expressed genes that correlate with behavioural variation across three $S$. carpocapsae strains. We have identified four srsx GPCR genes and a sodium transporter gene that correlate with enhanced chemotaxis of S. carpocpsae IJs. A neuropeptide-like protein gene, srt GPCR gene and sodium transporter gene are found to correlate with reduced nictation behaviour. Numerous microRNAs are predicted to target neuronal genes, and display expression profiles that correlate with behavioural variation. These data provide important insight to the transcriptional basis of host-finding behaviours in this species, highlighting a potential role for microRNAs and gene isoform variation. 


\section{Supplementary information}

Supplementary information accompanies this paper at https://doi.org/10. 1186/s12864-019-6179-y.

Additional file 1. List of all differentially expressed genes ( $>2 \log 2$ fold, $P<0.0001)$ across all pairwise strain comparisons.

Additional file 2. List of all differentially expressed genes represented in heatmaps, including annotated gene names, fold changes across pairwise comparisons, and adjusted $P$ values.

Additional file 3. List of S. carpocapsae microRNAs, sequences and read counts across strains.

Additional file 4. List of differentially expressed microRNAs (> $2 \log 2$ fold, $P<0.0001)$, including pairwise fold changes, and adjusted $P$ values.

Additional file 5. MicroRNA target predictions 1: Miranda output file for microRNA targets in 5'UTRs using default setting.

Additional file 6. MicroRNA target predictions 2: Miranda output file for microRNA targets in 5'UTRs using strict setting.

Additional file 7. MicroRNA target predictions 3: Miranda output file for microRNA targets in $3^{\prime} U T R s$ using default setting.

Additional file 8. MicroRNA target predictions 4: Miranda output file for microRNA targets in 3'UTRs using stricted setting.

Additional file 9. RSEM isoform quantification file for S. carpocapsae strains.

\section{Abbreviations}

FLP: FMRFamide-Like Peptide; GPCR: G-Protein Coupled Receptor; IJ: Infective Juvenile; INS: INSulin-like peptide; NLP: Neuropeptide-Like Protein;

UTR: UnTranslated Region

\section{Acknowledgements}

Waxworms infected with S. carpocapsae (Breton), were a gift from Prof Nelson Simões, University of the Azores, Portugal. S. carpocapsae (UK1) IJs were a gift from BASF. Microdirt chip PDMS moulds were a gift from Prof Junho Lee, Seoul National University, Korea. The authors would like to thank Matthew Sturrock and Steven Dyer for critical reading of the manuscript.

\section{Authors' contributions}

NDW, DC, CM and RM generated data and wrote the manuscript. JJD conceived the project, and wrote the manuscript. All authors read and approved the final manuscript.

\section{Funding}

NDW was funded by the Bill and Melinda Gates Foundation, DC was funded by a GCRF pilot grant from the NI Department for the Economy, CMcC was funded by a proof of concept grant from Invest NI, RM was funded by a PhD studentship from the QUB Business Alliance Fund. The funders had no role in experimental design or preparation of this manuscript. All authors have read and approved this manuscript.

\section{Availability of data and materials}

The S. carpocapsae strains used in this study are maintained within the Dalzell lab, and are freely available upon request. Illumina HiSeq reads for transcriptome and small RNAs across each S. carpocapsae strain are available within the SRA library under project code: PRJNA436466.

\section{Ethics approval and consent to participate}

Not applicable.

\section{Consent for publication}

Not applicable.

\section{Competing interests}

The authors declare that they have no competing interests.
Received: 28 November 2018 Accepted: 10 October 2019

Published online: 21 November 2019

\section{References}

1. Spencer SG, Hallem EA. Mechanisms of host seeking by parasitic nematodes. Mol Biochem Parasitol. 2016;208(1):23-32. https://doi.org/10. 1016/j.molbiopara.2016.05.007.

2. Castelletto ML, Gang SS, Okubo RP, Tselikova AA, Nolan TJ, Platzer EG, et al. Diverse host-seeking behaviors of skin-penetrating nematodes. PLoS Pathog. 2014;10(8):e1004305. https://doi.org/10.1371/journal.ppat.1004305.

3. Chaisson KE, Hallem EA. Chemosensory behaviors of parasites. Trends Parasitol. 2012;28(10):427-36. https://doi.org/10.1016/j.pt.2012.07.004.

4. Dillman AR, Guillermin ML, Lee JH, Kim B, Sternberg PW, Hallem EA. Olfaction shapes host-parasite interactions in parasitic nematodes. PNAS. 2012;109(35):E2324-E33. https://doi.org/10.1073/pnas.1211436109.

5. Ruiz F, Gang SS, Castelletto ML, Hallem EA. Experience-dependent olfactory behaviors of the parasitic nematode Heligmosomoides polygyrus. PLoS Pathog. 2017;13(11):e1006709. https://doi.org/10.1371/journal.ppat.1006709.

6. Mousley A, McVeigh P, Dalzell JJ, Maule AG. In: Kennedy MW, Harnett W, editors. ISBN: 9781845937591 Nematode neuropeptide communication systems. In, parasitic nematodes: molecular biology, biochemistry and immunology: CAB International; 2013. https://doi.org/10.1079/ 9781845937591.0279.

7. Peymen K, Watteyne J, Frooninckx L, Schoofs L, Beets I. The FMRFamide-like peptide family in nematodes. Front Endocrinol. 2014;5:90. https://doi.org/10. 3389/fendo.2014.00090.

8. Lee JS, Shih PY, Schaedel ON, Quintero-Cadena P, Rogers AK, Sternberg PW. FMRF amide-like peptides expand the behavioural repertoire of a densely connected nervous system. PNAS. 2017;114(50):E10726-35. https://doi.org/ 10.1073/pnas.1710374114.

9. Morris R, Wilson L, Sturrock M, Warnock ND, Carrizo D, Cox D, et al. A neuropeptide modulates sensory perception in the entomopathogenic nematode Steinernema carpocapsae. PLoS Pathog. 2017;13(3):e1006185. https://doi.org/10.1371/journal.ppat.1006185

10. Lee D, Lee $H$, Kim N, Lim DS, Lee J. Regulation of a hitchhiking behavior by neuronal insulin and TGF- $\beta$ signaling in the nematode Caenorhabditis elegans. Biochem Biophys Res Commun. 2017:484(2):323-30. https://doi.org/ 10.1016/j.bbrc.2017.01.113

11. Warnock ND, Wilson L, Patten C, Fleming CC, Maule AG, Dalzell Jر. Nematode neuropeptides as transgenic nematicides. PLoS Pathog. 2017; 13(2):e1006237. https://doi.org/10.1371/journal.ppat.1006237.

12. Hobert $\mathrm{O}$. The neuronal genome of Caenorhabditis elegans. WormBook: The C. elegans Research Community, WormBook; 2013. https://doi.org/10.1895/ wormbook.1.161.1.

13. Jovelin R, Cutter AD. Microevolution of nematode miRNAs reveals diverse modes of selection. Genome Biol Evol. 2014;6(11):3049-63. https://doi.org/ 10.1093/gbe/evu239.

14. Heimberg AM, Sempere LF, Moy VN, Donoghue PC, Peterson KJ. MicroRNAs and the advent of vertebrate morphological complexity. PNAS. 2008;105: 2946-50. https://doi.org/10.1073/pnas.0712259105.

15. Iwama H, Kato K, Imachi H, Murao K, Masaki T. Human microRNAs originated from two periods at accelerated rates in mammalian evolution. Mol Biol Evol. 2013;30:613-26. https://doi.org/10.1093/molbev/mss262.

16. Meunier J, Lemoine F, Soumillon M, Liechti A, Guschanski K, Hu H, Khaitovich $\mathrm{P}$, Kaessmann $\mathrm{H}$. Birth and expression evolution of mammalian microRNA genes. Genome Res. 2013;23(1):34-45. https://doi. org/10.1101/gr.140269.

17. Lee RC, Feinbaum RL, Ambros V. The C. elegans heterochronic gene lin-4 encodes small RNAs with antisense complementarity to lin-14. Cell. 1993; 75(5):843-54 DOI not available.

18. Konopka W, Kiryk A, Novak M, Herwerth M, Parkitna JR, et al. MicroRNA loss enhances learning and memory in mice. J Neurosci. 2010;30(44):14835-42. https://doi.org/10.1523/JNEUROSCI.3030-10.2010.

19. Ashraf SI, MCLoon AL, Sclarsic SM, Kunes S. Synaptic protein synthesis associated with memory is regulated by the RISC pathway in Drosophila. Cell. 2006;124(1):191-205. https://doi.org/10.1016/j.cell.2005.12.017.

20. Than MT, Kudlow BA, Han M. Functional analysis of neuronal MicroRNAs in Caenorhabditis elegans Dauer formation by combinational genetics and neuronal miRISC Immunoprecipitation. PLoS Genet. 2013;9(6):e1003592. https://doi.org/10.1371/journal.pgen.1003592. 
21. Lu D, Macchietto M, Chang D, Barros MM, Baldwin J, Mortazavi A, Dillman AR. Activated entomopathogenic nematode infective juveniles release lethal venom proteins. PLoS Pathog. 2017;13(4):31006302. https://doi.org/10.1371/ journal.ppat.1006302

22. Spence KO, Lewis EE, Perry RN. Host-finding and invasion by entomopathogeic and plant-parasitic nematodes: evaluating the ability of laboratory bioassays to predict field results. J Nematol. 2008;40(2):93-8 DOI not available.

23. Campbell JF, Kaya HK. How and why a parasitic nematode jumps. Nature. 1999:397:485-6. https://doi.org/10.1038/17254.

24. Lee H, Choi MK, Lee D, Kim HS, Hwang H, Kim H, Park S, Paik YK, Lee J. Nictation, a dispersal behavior of the nematode Caenorhabditis elegans, is regulated by IL2 neurons. Nat Neurosci. 2011;15(1):107-12. https://doi.org/ 10.1038/nn.2975

25. White G. A method for obtaining infective nematode larvae from cultures. Am Assoc Adv Sci. 1927;66(1709):302-3. https://doi.org/10.1126/science.66. 1709.302-a.

26. Lee $\mathrm{D}$, Lee $\mathrm{H}$, Choi M-k, Park S, Lee J. Nictation assays for Caenorhabditis and other nematodes. Bioprotocol. 2015;5:e1433. https://doi.org/10.21769/ BioProtoc. 1433.

27. Andrew, S. FastQC: a quality control tool for high throughput sequence data, 2010; http://www.bioinformatics.babraham.ac.uk/projects/fastqc

28. Bolger AM, Lohse M, Usadel B. Trimmomatic: a flexible trimmer for illumine sequence data. Bioinformatics. 2014;30(15):2114-20. https://doi.org/10.1093/ bioinformatics/btu170.

29. Trapnell C, Williams BA, Pertea G, Mortazavi A, Kwan G, et al. Transcript assembly and quantification by RNA-seq reveals unannotated transcripts and isoform switching during cell differentiation. Nat Biotechnol. 2010;28(5): 511-5. https://doi.org/10.1038/nbt.1621.

30. Dillman AR, Macchietto M, Porter CF, Rogers A, Williams B, Antoshechkin I, et al. Comparative genomics of Steinernema reveals deeply conserved gene regulatory networks. Genome Biol. 2015;16:200. https://doi.org/10.1186/ s13059-015-0746-6.

31. Dobin A, Davis CA, Schlesinger F, Drenkow J, Zaleski C, Jha S, Batut P, Chaisson M, Gingeras TR. STAR: ultrafast universal RNA-seq aligner. Bioinformatics. 2013; 29(1):15-21. https://doi.org/10.1093/bioinformatics/bts635.

32. Li B, Dewey CN. RSEM: accurate transcript quantification from RNA-Seq data with or without a reference genome. BMC Bioinformatics. 2011;12(1):323. https://doi.org/10.1186/1471-2105-12-323.

33. Leng N, Dawson JA, Thomson JA, Ruotti V, Rissman Al, Smits BM, Haag JD, Gould MN, Stewart RM, Kendziorski C. EBSeq: an empirical Bayes hierarchical model for inference in RNA-seq experiments. Bioinformatics. 2013;29(8): 1035-43. https://doi.org/10.1093/bioinformatics/btt087.

34. Team RDC. R: A language and environment for statistical computing. Vienna: R Foundation for Statistical Computing; 2008. ISBN 3-900051-07-0, URL http://www.R-project.org

35. Love Ml, Huber W, Anders S. Moderated estimation of fold change and dispersion for RNA-Seq data with DESeq2. Genome Biol. 2014;15(12):550. https://doi.org/10.1186/s13059-014-0550-8.

36. Neuwirth E. RColorBrewer: ColorBrewer Palettes. R package version 1.1-2, 2014; https://CRAN.R-project.org/package=RColorBrewer

37. Warnes GR, Bolker B, Bonebakker L, Gentleman R, Huber W et al. gplots: Various R Programming Tools for Plotting Data. R package version 3.0.1, 2016; http://CRAN.R-project.org/package=gplots

38. Gentleman R, Biocore. Geneplotter: graphics related functions for Bioconductor, 2016; R package version 1.52.0.

39. Kolde R. pheatmap: Pretty Heatmaps. R package version 1.0.8, 2015; http:// CRAN.R-project.org/package=pheatmap.

40. Martin M. Cutadapt removes adapter sequences from high-throughput sequencing reads. EMBnet J. 2011;17(1):10-2. https://doi.org/10.14806/ej.17.1.200

41. Friedländer MR, Mackowiak SD, Li N, Chen W, Rajewsky N. MiRDeep2 accurately identifies known and hundreds of novel microRNA genes in seven animal clades. Nucleic Acids Res. 2012;40(1):37-52. https://doi.org/ 10.1093/nar/gkr688

42. Howe KL, Bolt BJ, Cain S, Chan J, Chen WJ, Davis P, et al. WormBase 2016 : expanding to enable helminth genomic research. Nucleic Acids Res. 2015; 1217. https://doi.org/10.1093/nar/gkv1217.

43. Enright AJ, John B, Gaul U, Tuschl T, Sander C, Marks DS. MicroRNA targets in Drosphila. Genome Biol. 2003;5(1):R1. https://doi.org/10.1186/gb-2003-5-1-r1.

44. Thomas M, Lieberman J, Lal A. Desperately seeking microRNA targets. Nat Struct Mol Biol. 2010;17:1169-74. https://doi.org/10.1038/nsmb.1921.
45. Zisoulis DG, Lovci MT, Wilbert ML, Hutt KR, Liang TY, et al. Comprehensive discovery of endogenous Argonaute binding sites in Caenorhabditis elegans. Nat Struct Mol Biol. 2010;17:173-9. https://doi.org/10.1038/nsmb.1745.

46. Chen N, Harris TW, Antosheckin I, Bastiani C, Bieri T, et al. WormBase: a comprehensive data resource for Caenorhabditis biology and genomics. Nucleic Acids Res. 2005;33:D383-9. https://doi.org/10.1093/nar/gki066.

47. Dalzell JJ, McVeigh P, Warnock ND, Mitreva M, Bird DM, Abad P, et al. RNAi effector diversity in nematodes. PLoS Negl Trop Dis. 2011;5(6):e1176. https:// doi.org/10.1371/journal.pntd.0001176

48. McVeigh P, Kimber MJ, Novozhilova E, Day TA. Neuropeptide signalling systems in flatworms. Parasitology. 2005;131:S41-55. https://doi.org/10.1017/ S0031182005008851.

49. Rougon-Cardoso A, Flores-Ponce M, Ramos-Aboites HE, Martinez-Guerrero CE, Hao Y-J, et al. The genome, transcriptome, and proteome of the nematode Steinernema carpocapsae: evolutionary signatures of a pathogenic lifestyle. Sci Rep. 2016;6:37536. https://doi.org/10.1038/srep37536.

50. Cook DE, Zdraljevic S, Roberts JP, Adersen EC. CeNDR, the Caenorhabditis elegans natural diversity resource. Nucleic Acids Res. 2017;45(D1):D650-7. https://doi.org/10.1093/nar/gkw893.

51. Lee D, Yang H, Kim J, Brady S, Zradljevic S, Zamanian M, Kim H, Paik YK, Kruglyak L, Andersen EC, Lee J. The genetic basis of natural variation in a phoretic behaviour. Nat Commun. 2017;8(1):273. https://doi.org/10.1038/ s41467-017-00386-x.

52. Coburn CM, BArgman Cl. A putative cyclic nucleotide-gated channel is required for sensory development and function in C. elegans. Neuron. 1996; 17:695-706. https://doi.org/10.1016/S0896-6273(00)80201-9.

53. Coburn CM, Mori I, Ohshima Y, BArgmann Cl. A cyclic nucleotide-gated channel inhibits sensory axon outgrowth in larval and adult Caenorhabditis elegans: a distinct pathway for maintenance of sensory axon structure. Development. 1998;125:249-58 No doi available.

54. Gruner M, Nelson D, Winbush A, Hintz R, Ryu L, Chung SH, Kim K, Gabel CV, van der Linden AM. Feeding state, insulin and NPR-1 modulate chemoreceptor gene expression via integration of sensory and circuit inputs. PLOS Genet. 2014;10(10):e1004707. https://doi.org/10.1371/journal.pgen.1004707.

55. Wang XD, Ishibashi N. Infection of the entomopathogenic nematode, Steinernema carpocasae, as affected by the presence of Steinernema glaseri. J Nematol. 1999;31(2):207-11 DOI not available.

56. Lee JH, Dillman AR, Hallem EA. Temperature-dependent changes in the host-seeking behaviors of parasitic nematodes. BMC Biol. 2016;14(1):1. https://doi.org/10.1186/s12915-016-0259-0.

57. Okumura $\mathrm{E}$, Yoshiga T. Host orientation using volatiles in the phoretic nematode Caenorhabditis japonica. J Exp Biol. 2014;217(18):3197-9. https:// doi.org/10.1242/jeb.105353.

58. Yemini El, Jucikas T, Grundy LJ, Brown AE, Schafer WR. A database of Caenorhabditis elegans behavioural phenotypes. Nat Methods. 2013;10:8779. https://doi.org/10.1038/nmeth.2560.

59. Gillan V, Maitland K, Laing R, Gu H, Marks ND, et al. Increased expression of a microRNA correlates with anthelmintic resistance in parasitic nematodes. Front Cell Infect Microbiol. 2017. https://doi.org/10.3389/fcimb.2017.00452.

60. Gu HY, Marks ND, Winter AD, Weir W, Tzelos T, McNeilly TN, Britton C, Devaney E. Conservation of a microRNA cluster in parasitic nematodes and profiling of miRNAs in excretory-secretory products and microvesicles of Haemonchus contortus. PLoS Negl Trop Dis. 2017;11(11):e0006056. https:// doi.org/10.1371/journal.pntd.0006056.

61. Zamanian M, Cook DE, Zdraljevic S, Brady SC, Lee D, Lee J. Discovery of unique loci that underlie nematode responses to benzimidazoles. BioRxiv. 2017. https://doi.org/10.1101/116970.

62. Pinzón N, Blaise L, Martinez L, Sergeeva A, Presumey J, Apparailly F, Seitz H. MicroRNA target prediction programs predict many false positives. Genome Res. 2017;27(2):234-45. https://doi.org/10.1101/gr.205146.116.

63. Mangone M, Manoharan AP, Thierry-Mieg D, Thierry-Mieg J, Han T, et al. The landscape of C. elegans 3'UTRs. Science. 2010;329(5990):432-5. https:// doi.org/10.1126/science.1191244

64. Blazie SM, Geissel HC, Wilky H, Joshi R, Newbern J, Mangone M. Alternative polyadenylation directs tissue-specific miRNA targeting in Caenorhabditis elegans somatic tissues. Genetics. 2017;206(2):757-74. https://doi.org/10. 1534/genetics.116.196774.

65. Gustafsson C, Vallverdú J. The best model of a cat is several cats. Trends Biotechnol. 2016;34(3):207-13. https://doi.org/10.1016/j.tibtech.2015.12.006.

66. Macchietto M, Angdembey D, Heidarpour N, Serra L, Rodriguez B, El-Alli N, Mortazavi A. Comparative transcriptomics of Steinernema and Caenorhabditis 
single embryos reveals orthologous gene expression convergence during late embryogenesis. Genome Biol Evol. 2017;9(10):2681-96. https://doi.org/ 10.1093/gbe/evx195

67. Choe A, von Reuss SH, Kogan D, Gasser RB, Platzer EG, Schroeder FC, Sternberg PW. Ascaroside signalling is widely conserved among nematodes. Curr Biol. 2012;22(9):772-80. https://doi.org/10.1016/j.cub.2012.03.024.

68. Blaxter M, Koutsovoulos $\mathrm{G}$. The evolution of parasitism in Nematoda. Parasitology. 2015;142(Suppl 1):S26-39. https://doi.org/10.1017/ S0031182014000791

69. Lu D, Baiocchi T, Dillman AR. Genomics of entomopathogenic nematodes and implications for pest control. Trends Parasitol. 2016;32(8):588-98. https://doi.org/10.1016/j.pt.2016.04.008.

\section{Publisher's Note}

Springer Nature remains neutral with regard to jurisdictional claims in published maps and institutional affiliations.

Ready to submit your research? Choose BMC and benefit from:

- fast, convenient online submission

- thorough peer review by experienced researchers in your field

- rapid publication on acceptance

- support for research data, including large and complex data types

- gold Open Access which fosters wider collaboration and increased citations

- maximum visibility for your research: over $100 \mathrm{M}$ website views per year

At BMC, research is always in progress.

Learn more biomedcentral.com/submissions 